\title{
Exposure to blue light during lunch break: effects on autonomic arousal and behavioral alertness
}

\author{
Emi Yuda, Hiroki Ogasawara, Yutaka Yoshida and Junichiro Hayano* (D)
}

\begin{abstract}
Background: Exposures to melanopsin-stimulating (melanopic) component-rich blue light enhance arousal level. We examined their effects in office workers.

Main body of abstract: Eight healthy university office workers were exposed to blue and orange lights for 30 min during lunch break on different days. We compared the effects of light color on autonomic arousal level assessed by heart rate variability (HRV) and behavioral alertness by psychomotor vigilance tests (PVT). Heart rate was higher and high-frequency $(\mathrm{HF}, 0.150 .45 \mathrm{~Hz})$ power of HRV was lower during exposure to the blue light than to orange light. No significant difference with light color was observed, however, in any HRV indices during PVT or in PVT performance after light exposure.

Short conclusion: Exposure to blue light during lunch break, compared with that to orange light, enhances autonomic arousal during exposure, but has no sustained effect on autonomic arousal or behavioral alertness after exposure.
\end{abstract}

Keywords: Alertness, Arousal, Blue light, Heart rate variability, Melanopsin, Non-image forming vision, Orange light, Organic light-emitting diode, Psychomotor vigilance

\section{Background}

Along with the development of lighting elements capable of various color-rendering, the introduction of color lighting in life environment is taking on reality. Such lightings may be used for adjusting our mind and physical states to suit our desired activities, such as work, study, rest, and sleep [1-4]. In previous studies [5, 6], we have demonstrated that exposure to melanopsinstimulating (melanopic) component-rich blue light enhances autonomic arousal and behavioral alertness. These observations, however, were obtained in laboratory settings. In this study, we examined the effects of exposure to blue light or orange lights during the lunch break on autonomic arousal and behavioral alertness in office workers.

\footnotetext{
*Correspondence: hayano@med.nagoya-cu.ac.jp

Department of Medical Education, Nagoya City University Graduate School of Medical Sciences, 1 Kawasumi Mizuho-cho Mizuho-ku, Nagoya 467-8601, Aichi, Japan
}

(c) The Author(s). 2017 Open Access This article is distributed under the terms of the Creative Commons Attribution 4.0 International License (http://creativecommons.org/licenses/by/4.0/), which permits unrestricted use, distribution, and reproduction in any medium, provided you give appropriate credit to the original author(s) and the source, provide a link to the Creative Commons license, and indicate if changes were made. The Creative Commons Public Domain Dedication waiver (http://creativecommons.org/publicdomain/zero/1.0/) applies to the data made available in this article, unless otherwise stated.

\section{Main text}

\section{Subjects and protocols}

We studied eight healthy university office workers (mean age $\pm S D, 27 \pm 7$ years, range $21-39$ years, 2 females) with normal color vision who were not taking any medications for $>2$ weeks and displayed a normal sinus rhythm on electrocardiogram (ECG).

We compared the effects on heart rate variability (HRV) and psychomotor vigilance test (PVT) caused by exposures to blue and orange lights during the lunch break in workplace. We prepared a light chamber in the workplace with an organic light-emitting diode (OLED) ceiling light system with adjustable color and brightness (Fig. 1), whose details have been reported elsewhere [6]. Table 1 shows the optical characteristics of blue and orange lights.

Experiments with the two light colors were performed on separated days with an interval of 5-7 days for washout. The color orders were counterbalanced across subjects. On each experimental day, Holter ECG was recorded continuously during experiment. Subjects took a light lunch 


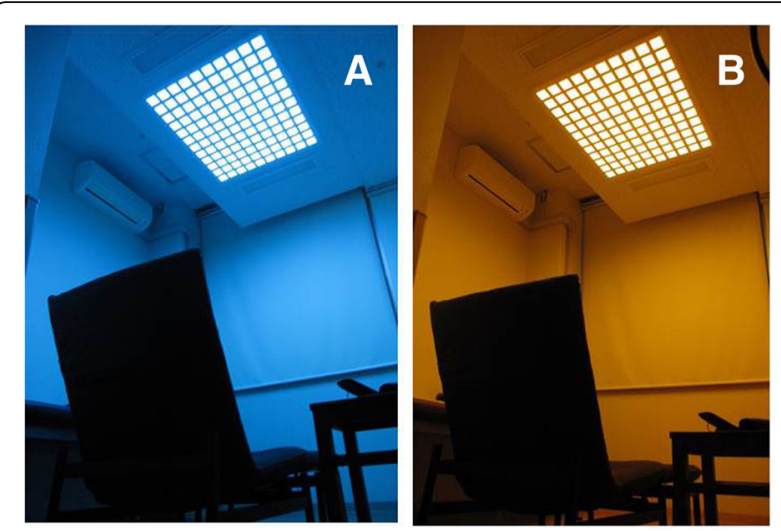

Fig. 1 Ceiling light chamber with organic light-emitting diode (OLED) when the lighting is $\mathbf{a}$ blue and $\mathbf{b}$ orange

(the same menu on two experimental days) with a drink not containing caffeine or alcohol at the university cafeteria. During the last $30 \mathrm{~min}$ of lunch break, they were exposed to blue or orange light in the light chamber (airconditioned at $24 \pm 2{ }^{\circ} \mathrm{C}$ ), sitting in a low chair with $60^{\circ}$ backrest just below the ceiling light.

After the lunch break, a 5-min PVT was performed in their work office which was illuminated by fluorescent lamps with a correlated color temperature of $4010 \mathrm{~K}$, an illuminance of $450 \mathrm{~lx}$, a photon flux density of $6.7 \mu \mathrm{mol} / \mathrm{m}^{2} / \mathrm{s}$, and a relative melanopic component of $20.4 \%$. PVT was performed with validated software (PC-PVT) [7]. The detailed method for PVT has been reported elsewhere [6]. The anticipation was set at $100 \mathrm{~ms}$, deadline at $65,000 \mathrm{~ms}$, minor lapse at $500 \mathrm{~ms}$, and total trial time at $300 \mathrm{~s}$.

\section{Data analysis}

$\mathrm{R}-\mathrm{R}$ interval time series obtained from Holter ECG were extracted for the durations of OLED light exposure (30 min) and PVT (5 min) for the analysis of HRV. HRV was analyzed by the methods reported elsewhere $[8,9]$.

Table 1 Characteristics of organic light-emitting diode (OLED) lights used in this study

\begin{tabular}{lll}
\hline & Blue & Orange \\
\hline Illuminance, Ix & 12.9 & 17.7 \\
Irradiance, $\mu \mathrm{W} / \mathrm{cm}^{2}$ & 8.02 & 6.54 \\
Chromaticity $(x, y)$ & $0.14,0.16$ & $0.56,0.42$ \\
Weighted mean wavelength, $\mathrm{nm}$ & 485 & 622 \\
$\mathrm{PFD}, \mu \mathrm{mol} / \mathrm{m}^{2} / \mathrm{s}$ & 0.323 & 0.333 \\
Melanopic PFD, $\mu \mathrm{mol} / \mathrm{m}^{2} / \mathrm{s}^{\mathrm{a}}$ & 0.225 & 0.026 \\
Relative melanopic content, \% & 69.6 & 7.9 \\
\hline
\end{tabular}

PFD photon flux density

${ }^{a}$ Calculated from melanoptic spectral efficiency adjusted for human prereceptoral filtering $[13,14]$
The power of the low-frequency (LF, $0.04-0.15 \mathrm{~Hz}$ ) and the high-frequency (HF, $0.15-0.40 \mathrm{~Hz})$ components were computed by fast Fourier transformation. We used heart rate as an index of sympatho-vagal balance, HF power as cardiac vagal function, and LF-to-HF power ratio (LF/ HF) as sympathetic predominance in cardiac autonomic functions. The power of LF and HF components was transformed into natural logarithmic value.

PVT performance was analyzed according to the earlier studies [10]. We measured the number of minor lapses (reaction time (RT) $\geq 500 \mathrm{~ms}$ ) with transformation $(\sqrt{x}+\sqrt{x+1})$, fastest and slowest 10th percentile RTs, and difference between fastest and slowest RTs.

We used the Statistical Analyses System version 9.4 (SAS institute Inc., Cary, NC, USA) for statistical analysis. To examine the effects of light color on HRV indices and PVT performance, we compared the values between blue and orange lights with paired $t$ test. $P<0.05$ was considered to be statistically significant.

\section{Results}

The HRV indices during exposures to blue and orange lights and during PVT after exposure in individual subjects are shown in Fig. 2. Heart rate was higher $(P=0.001)$ and HF power was lower $(P=0.03)$ during exposure to blue light than to orange light, whereas there was no significant difference with light color in any HRV indices during PVT after exposure. As shown in Fig. 3, no

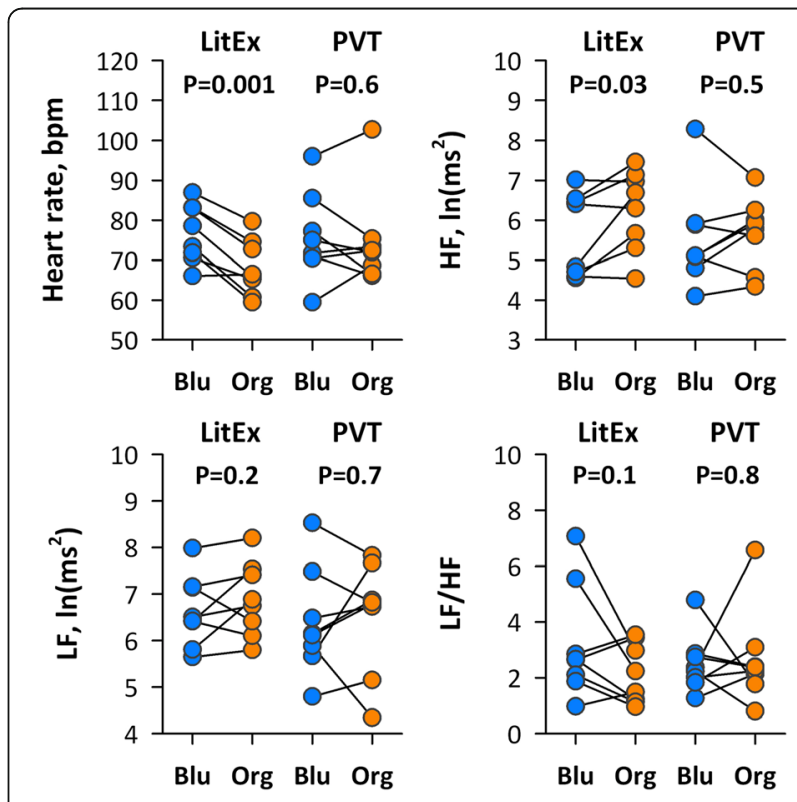

Fig. 2 Differences between blue and orange lights in heart rate and heart rate variability (HRV) indices during light exposure (LitEx) and psychomotor vigilance test (PVT) after exposure in individual subjects $(n=8)$. Blu blue light, HF high-frequency component, LF low-frequency component, LF/HF LF-to-HF ratio in power, Org orange light 


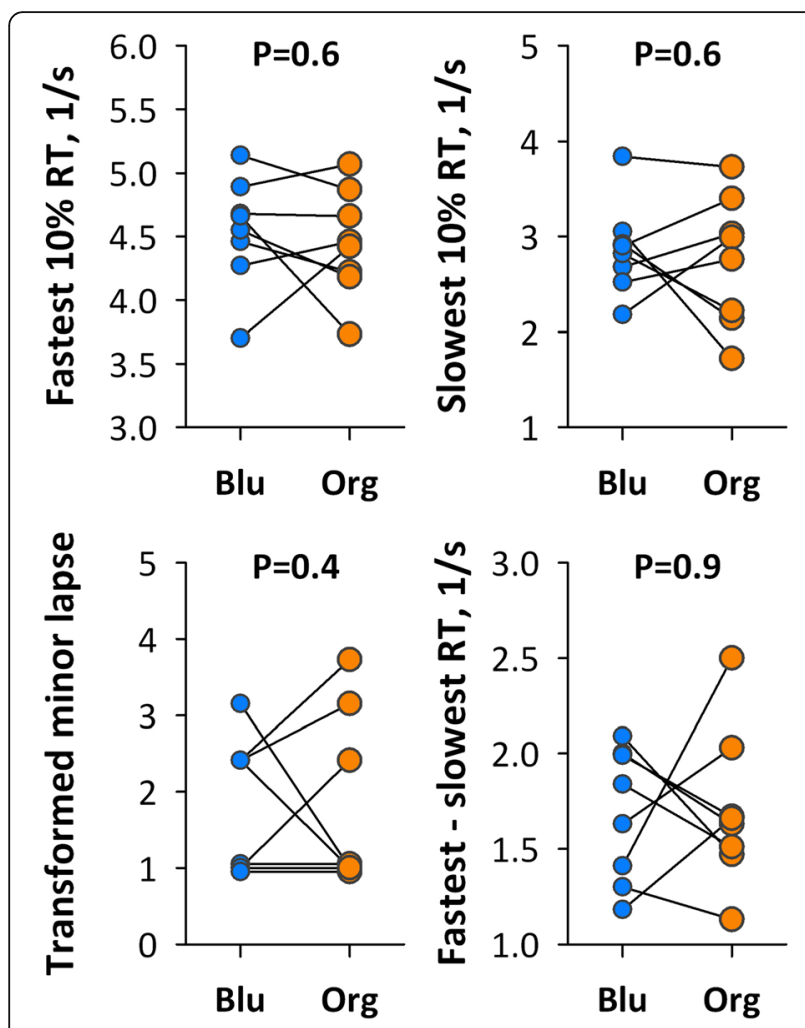

Fig. 3 Differences between blue and orange lights in the performance of PVT after light exposure in individual subjects $(n=8)$. Blu blue light, Org orange light, $R T$ reaction time

significant difference with light color was observed in any indices of PVT performance after exposure.

\section{Conclusions}

From these findings, we conclude that compared with orange light, blue light during lunch break enhances autonomic arousal during exposure, but causes no sustained differences in autonomic arousal or behavioral alertness after exposure.

In this study, we observed higher heart rate and lower HF power during exposure to blue light than orange light. These observations seem consistent with earlier studies suggesting autonomic arousal effects of melanopic component-rich blue light [1-6]. In contrast, we failed to detect significant difference with light color in autonomic indices during PVT or PVT performed after exposure. This seems inconsistent with those of an earlier study of Chellappa et al. [2] and of our previous study [6]. We performed PVT $30 \mathrm{~min}$ after light exposure in this study, while it was performed during light exposure in Chellappa's study [2] and $<5$ min after exposure in our previous study [6]. The difference may be attributable to the duration of effects. In any case, however, exposure to blue light during lunch break may not cause substantial difference from orange light in behavioral alertness during afternoon's work.
This study has several limitations. First, this study was performed in university office workers. Our observations may not be generalized for other types of workers. Second, the intensity of blue light we used was low (PFD, $\left.0.323 \mu \mathrm{mol} / \mathrm{m}^{2} / \mathrm{s}\right)$, but the effects may differ depending on light intensity $[11,12]$. Third, because we did not measure melatonin secretion, we were unable to determine whether the light exposures affect the entrainment to environmental light-dark cycles or not.

\section{Abbreviations}

ECG: Electrocardiogram; HF: High frequency; HRV: Heart rate variability; LF: Low frequency; LF/HF: LF-to-HF ratio; OLED: Organic light-emitting diode: PFD: Photon flux density; PVT: Psychomotor vigilance test; RT: Reaction time

\section{Acknowledgements}

This study was performed as a part of the collaborative studies with

Chemical materials Evaluation and Research Base (CEREBA), Japan.

\section{Funding}

This study was supported by the New Energy and Industrial Technology Development Organization (NEDO), Japan.

\section{Availability of data and materials}

All data described in this study are freely available to any scientist wishing to use them in a way that ensures the participant's confidentiality. The software described in this study is also available to any scientist wishing to use them. In any case, the data and software described in the manuscript are available for testing by reviewers in a way that preserves the reviewers' anonymity.

\section{Authors' contributions}

EY participated in the design and coordination of the study and drafted the manuscript. $\mathrm{HO}$ developed the software for the study and participated in the coordination of the study. YY participated in the data analysis and performed the statistical analysis. JH conceived the study, interpreted the results, and helped to draft the manuscript. All authors read and approved the final manuscript.

\section{Ethics approval and consent to participate}

The present study was performed according to the protocol that was approved by the Institutional Review Board of Nagoya City University Graduate School of Medical Sciences and Nagoya City University Hospital (No. 60160164). All the subjects of this study gave their written informed consent to participate in this study.

\section{Consent for publication}

I assure you that all the authors have read the manuscript and approved its submission to the Journal of Physiological Anthropology.

\section{Competing interests}

The authors declare that they have no competing interests.

\section{Publisher's Note}

Springer Nature remains neutral with regard to jurisdictional claims in published maps and institutional affiliations.

Received: 28 May 2017 Accepted: 5 July 2017

Published online: 11 July 2017

\section{References}

1. Cajochen C, Munch M, Kobialka S, Krauchi K, Steiner R, Oelhafen P, Orgul S, Wirz-Justice A. High sensitivity of human melatonin, alertness, thermoregulation, and heart rate to short wavelength light. J Clin Endocrinol Metab. 2005;90(3):1311-6.

2. Chellappa SL, Steiner R, Blattner P, Oelhafen P, Gotz T, Cajochen C. Nonvisual effects of light on melatonin, alertness and cognitive performance: can blue-enriched light keep us alert? PLoS One. 2011;6(1):e16429. 
3. Choi CJ, Kim KS, Kim CM, Kim SH, Choi WS. Reactivity of heart rate variability after exposure to colored lights in healthy adults with symptoms of anxiety and depression. Int J Psychophysiol. 2011;79(2):83-8.

4. Daneault V, Hebert M, Albouy G, Doyon J, Dumont M, Carrier J, Vandewalle $\mathrm{G}$. Aging reduces the stimulating effect of blue light on cognitive brain functions. Sleep. 2014;37(1):85-96.

5. Yuda E, Ogasawara H, Yoshida Y, Hayano J. Suppression of vagal cardiac modulation by blue light in healthy subjects. J Physiol Anthropol. 2016;35:24.

6. Yuda E, Ogasawara H, Yoshida Y, Hayano J. Enhancement of autonomic and psychomotor arousal by exposures to blue wavelength light: importance of both absolute and relative contents of melanopic component. J Physiol Anthropol. 2017;36(1):13.

7. Khitrov MY, Laxminarayan S, Thorsley D, Ramakrishnan S, Rajaraman S, Wesensten NJ, Reifman J. PC-PVT: a platform for psychomotor vigilance task testing, analysis, and prediction. Behav Res Methods. 2014:46(1):140-7.

8. Camm AJ, Malik M, Bigger Jr JT, Breithardt G, Cerutti S, Cohen RJ, Coumel P, Fallen EL, Kleiger RE, Lombardi F, Malliani A, Moss AJ, Rottman JN, Schmidt G, Schwartz PJ, Singer DH. Task Force of the European Society of Cardiology and the North American Society of Pacing and Electrophysiology. Heart rate variability: standards of measurement, physiological interpretation and clinical use. Circulation. 1996;93(5):1043-65.

9. Hayano J. Introduction to heart rate variability. In: Iwase S, Hayano J, Orimo S, editors. Clinical assessment of the autonomic nervous system. Japan: Springer; 2016. p. 109-27.

10. Graw P, Krauchi K, Knoblauch V, Wirz-Justice A, Cajochen C. Circadian and wake-dependent modulation of fastest and slowest reaction times during the psychomotor vigilance task. Physiol Behav. 2004;80(5):695-701.

11. Kozaki T, Koga S, Toda N, Noguchi H, Yasukouchi A. Effects of short wavelength control in polychromatic light sources on nocturnal melatonin secretion. Neurosci Lett. 2008;439(3):256-9.

12. Brainard GC, Hanifin JP, Warfield B, Stone MK, James ME, Ayers M, Kubey A, Byrne $B$, Rollag M. Short-wavelength enrichment of polychromatic light enhances human melatonin suppression potency. J Pineal Res. 2015;58(3):352-61.

13. Enezi J, Revell V, Brown T, Wynne J, Schlangen L, Lucas R. A "melanopic" spectral efficiency function predicts the sensitivity of melanopsin photoreceptors to polychromatic lights. J Biol Rhythms. 2011;26(4):314-23.

14. Price LLA. Report on the First International Workshop on Circadian and Neurophysiological Photometry, 2013. International Commission on Illumination, 2015 CIE TN 003. 2015.

\section{Submit your next manuscript to BioMed Central and we will help you at every step:}

- We accept pre-submission inquiries

- Our selector tool helps you to find the most relevant journal

- We provide round the clock customer support

- Convenient online submission

- Thorough peer review

- Inclusion in PubMed and all major indexing services

- Maximum visibility for your research

Submit your manuscript at www.biomedcentral.com/submit

CBiomed Central 